\title{
CONTRIBUCION DE LA ARQUEOLOGIA MEDICA AL ESTUDIO DE LAS RUINAS DE LAS MISIONES JESUITICO-GUARANIES DE ARGENTINA, BRASIL Y PARAGUAY
}

Silvio Palacios*

\section{JUSTIFICACIÓN}

En el contenido multIdisciplinario de la investigación arqueológica, la arqueología médica ocupa una posición de estudio complementario, objetivo, científico y susceptible de interpretación.

En el tema misionero jesuítico-guaraní existe un predominio de los estudios históricos y en menor proporción los antropológicos y arqueológicos. Todavía hay dificultades para avanzar y completar el conocimiento a través de pruebas y experiencias objetivas y controladas.

Si es posible hacer dataciones y diagnósticos con huesos, coprolitos y otros vestigios de procedencia humana, de 500, 2000, 5000 y muchos más años, no debieran existir dificultades importantes con el examen de material cuyo origen se remonta a un período de 200 a 400 años, como máximo, o sea durante la existencia organizada de los treinta pueblos misioneros

* Pesquisador espanhol, PhD, MD, DTM.

Estudos Ibero-Americanos. PUCRS, v.XX, n.2, p. 45-64, dezembro, 1994 
jesuítico-guaraníes, en los siglos XVII y XVIII, 159 años desde 1609 fundación del primer pueblo misionero hasta 1768, año de la expulsión de los jesuítas, 383 años desde 1609 a 1992 y 224 años de 1768 a 1992.

Las investigaciones arqueológicas que se realizan actualmente en las ruinas de los pueblos misioneros jesuítico-guaraníes de Argentina, Brasil y Paraguay, pueden complementarse con métodos modernos de diagnóstico y contribuir a los estudios antropológicos y arqueológicos y a um mejor conocimiento sobre la "salud y enfermedad" de los habitantes de aquellas misiones.

En las investigaciones arqueológicas se ha concedido mayor prioridad al estudio de la arquitectura, los metales y la cerámica, que al estudio de los "huesos" y otros vestigios. Es probable que esto suceda por disponer de un material más abundante y de mejor accesibilidad y conservación.

Sin la participación de disciplinas importantes como la antropología, la arqueología y la medicina, sin un conocimiento de tradiciones, costumbres y "salud", un estudio histórico resultará incompleto.'La arqueología médica debe complementar la investigción histórica para permitir una interpretación más adequada del pasado.

Cuando se estudian muestras arqueológicas de huesos humanos y de otros vestigios, todavía persisten muchas incógnitas por despejar en la posible relación entre lesiones óseas y su etiología, por ejemplo. Este tipo de investigaciones no son fáciles y el comienzo habrá que definirlo como un "camino de acercamiento", hecho por etapas de "resultados parciales", pero esas son las razones más importantes para justificar, impulsar y estimular esos estudios.

\section{POSIBILIDADES DE EJECUCIÓN}

La característica de urbanización de los pueblos misioneros jesuíticoguaraníes, con la localización, generalmente uniforme, de la casa de los Padres, el Templo y el Cementerio, el número de habitantes, los años de existencia, la mortalidad comprobada y la seguridad que la gran mayoría eran enterrados en el cementerio siguiendo el rito católico, el número de esqueletos, de muestras óseas y otros vestigios, podría estar asegurada.

La conexión de los resultados de estos hallazgos y exámenes, con los registros históricos disponibles, facilitaría su localización, datación e interpretación. 
Existe una relativa facilidad para localizar los cementerios de algunos pueblos misioneros jesuítico-guaraníes, analizando los trazos urbanos que figuran en los Planos conocidos, por ejemplo, de San Miguel y San Juan en Brasil, San Ignacio Mini y San Carlos (que conservan restos de los muros de los cementerios) y Candelaria en Argentina.

Los tres países comprendidos, Argentina, Brasil y Paraguay, tienen posibilidades de ejecución porque disponen de Instituciones responsables y especialistas de reconocido prestigio que pueden cubrir la condición multidisciplinaria de las investigaciones, asi como Laboratorios Clinicos Universitarios de calidad, dnde ya realizan las técnicas incluídas en esta Contribuición.

Con frecuencia conviene solicitar ayudas oficiales o privadas, nacionales e internacionales, así como de especialistas de Universidades, familiarizados con este tipo de estudios.

Lo más acertado sería conseguir la ejecución "simultánea" por lo menos en un sitio arqueológico misionero de cada uno de los tres países. En forma simultánea y regidos por los mismos objetivos y técnicas selecceionadas.

\section{SUGERENCIAS}

Este Estudio no puede considerarse como un trabajo "aislado", sino incorporado en las actividades arqueológicas programadas actualmente en cada pueblo misionero jusuítico-guaraníe donde se efectúan las investigaciones.

Las discusiones, análisis e interpretación de la información obtenida deberá relacionarse, muy especialmente, con los conocimientos históricos, arqueológicos y antropológicos ya conocidos.

Es importante saber si coincide, o no, el cementerio del pueblo misionero objeto de esta investigación, con el lugar usado para el mismo fín, en épocas posteriores y actuales.

Habrá que solucionar les posibles limitaciones de orden juridica, de propiedad privada y eclesiástica que pueden dificultar la realización de los trabajos, obteniendo las respectivas autorizaciones. Parece que los cementerios de las ruinas de los pueblos mencionados anteriormente, ya hace mucho tiempo que no se destinan a esa finalidad. 
Si existieran limitaciones técnicas y/o financieras podrían seleccionarse los sitios arqueológicos y las técnicas que presenten mayor accesibilidad, economía y posibilidades de ejecución.

Esta investigación presupone un trabajo y un intercambio de ideas, principalmente entre historiadores, arqueólogos, arquitectos, antropológos y médicos especializados en las técnicas a realizarse.

Una buena coordinación podrá garantizar la calidad, disminuir el coste y el tiempo de ejecución.

El planteamiento y metodǫlogía de esto Estudio no tiene limites precisos y hay que concederle la flexibilidad, amplitud y profundidad que otorga el conocimiento y la imaginación de los investigadores.

\section{TÉCNICAS FACTIBLES DE APLICACIÓN}

En este Estudio, como punto de partida, podría pensarse en la obtención de muestras óseas humanas y vestigios que hagan sospechar materias fecales desecadas. La observación directa o macroscópica, la radiología y la microscopía constituirían el primer paso del examen de los huesos así como exámenes parasitológicos de las heces, posteriormente y de acuerdo con los especialistas y la experiencia acumulada, se estudiaría la realización de exámenes más complejos. Una relación de esos posibles metodos, sería la seguiente: radiografía, microscopía de rutina y eletrónica, PIAF-Prueba Indirecta de Anticuerpo Fluorescente -, ELISA-Enzyme linked Inmunosorbent Assay -, IRMA-Antígeno marcado con isótopo radioactivo -, ADNAcido desoxirribonucléico -, Prasitarias y Grupos Sanguíneos.

Lógicamente la selección de cada método o prueba requiere tener una idea clara de lo que se pretende para discutirla con el especialista. Para ello es fundamental que despues de haber llegado a un diagnóstico de sospecha por el estudio e interpretación de la información histórica, necesitemos una confirmación. También puede encararse, por curiosidad o requerimiento científico, aunque no se conozcan los antecedentes históricos, el saber si existen anormalidades en el material arqueológioo, sin condicionamientos ni sospechas o hipótesis previas.

En diversos sitios arqueológicos de América del Sur se han realizado, y se realizan, estudios aplicando técnicas modernas de diagnóstico para determinar, con la mayor precisión posible, las enfermedades que padecieron las poblaciones indígenas precolombinas, la dieta, sexo, edad y otras características anatómicas y de desarrollo. 
Un breve comentario sobre la determinación del "sexo" y "edad", que se verá facilitada o dificultada según se encuetra el material óseo: en esqueleto completo, hueso completo o fragmento. Para la determinación del sexo utilizan, con mayor frecuencia, el examen de las características morfológicas y métricas de la pelvis, aunque también existen diferencias en otros huesos. Con la determirnación de la edad hay más posibilidades, siendo las principales: las características de las suturas craneanas, el desarrollo epifisario y de los dientes y el examen histológico y radiográfico. La Facultad de Odontología podrá resolver las dudas que se presenten en las piezas dentarias.

Ya se aclaró que si es posible hacer diagnósticos retrospectivos con vestigios humanos de miles de años no se presentarán serios problemas para hacerlos en muestras de 200 a 400 años.

\section{FASES DE LA EJECUCIÓN TÉCNICA}

Para dar consistencia a esta Contribución, aquí se mencionan solamente algunos aspectos de excavaciones y otros, sin entrar en detalles científicos y técnicos propios del trabajo específico del arqueólogo. Tampoco se desarrolla la metodología de las llamadas "técnicas modernas" por ser propias de los médicos especialistas, y de su ejecución e interpretación de resultados.

\section{OBTENCIÓN DE MUESTRAS}

El empleo del material de las antiguas construcciones para casas y caminos, las excavaciones de buscadores de tesoros inexistentes, los procedimientos agrícolas que pueden destruir la estratificación, las nuevas urbanizaciones y los recursos insuficientes, son los principales obstáculos encontrados por los arqueólogos en sus actuales trabajos de campo, en las ruinas de los pueblos misioneros jesuítico-guaraníes.

En los restos óseos encontrados hasta ahora, en las excavaciones figuran, casi exclusivamente, los de procedencia animal, hallados junto a lugares de preparación de alimentos o en otros sitios demarcados como áras da trabajo. Estas muestras óseas encontradas en algunas excavaciones son numerosas y han sido objeto de buenos estudios de identificación y cuantificación, pero, como ya fue mencionado, su procedencia es de ganado 
vacuno. Es oportuno recordar el gran desarrollo ganadero, de distribución organizada y de consumo de carne en estas misiones.

De no existir dificultades do tipo religioso o jurídico, el trabajo especializado del arqueólogo, con el empleo del método de los "cuadrantes" - resultado de los exámenes morfológico y estratigráfico - facilitará, con bastante precisión, de qué extrato o nivel procede cada fragmento óseo encontrado. Es de esperar que cuando las excavaciones alcancen ciertos niveles, el arqueólogo podrá determinar, inicialmente, un diagnóstico de sospecha de la "base" del terreno utilizado como cementerio misionero y estar en condiciones de descartar el material óseo encontrado, más o menos superficialmente, y posibilitado de seleccionar las muestras que serán objeto del estudio específico de este Proyecto.

Probablemente existirá una distribuición característica de los esqueletos procedentes de cuerpos enterrados durante el período misionero jesuítico, e incluso tambén, que esa característica determine una mayor concentración de muestras óseas que pueden interpretarse como resultado de epidemias que se presentaron con alta mortalidad.

La localización de cementerios sería lo más importante pero, ocasionalmente, también algunos lugares de los templos misioneros porque allí enterraban caciques y otras autoridades guaraníes como figuran en lápidas sepulcrales, por ejemplo en las ruinas del templo del pueblo misionero de San Ignacio Mini, de Argentina. Conviene recordar la posibilidad de que los misioneros jesuítas fueran enterrados al pié del altar mayor, y a ciertas autoridades guaraníes en la parte posterior del piso de las naves de los templos.

Las muestras óseas humanas extraídas en las excavaciones deben ser representativas de los diferentes niveles del terreno estudiado - estratigrafía horizontal y vertical - para establecer una cronología hasta encontrar la "base".

La localización y el trabajo en el cementerio misionero facilitará las actividades de campo y de laboratorio porque lo probable será encontrar concentración de muestras óseas humanas sin requerir ampliar mucho el área en estudio. Si se localiza la "base" allí estarán las muestras correspondisntes a los primeros indios enterrados y primeros años del lugar destinado a cementerio. Por tratarse de un lugar "elegido", específico de enterramientos de la época misionera, es probable que no aparezcan restos óseos humanos pre-jesuíticos. El conocimiento de las fechas de fundación de cada pueblo misionero y fecha "tope" de 1768 , año de la expulsión de los jesuítas, 
facilitará una interpretación cronológica con la referencia de la datación de las muestras óseas seleccionadas, apoyada en los registros históricos.

Los huesos de origen humano, de los cementerios jusuítico-guaraníes no son solamente unos datos arqueológicos aislados, sino que habrá que considerarlos formando parte del contexto arqueológico del pueblo misionero y asociarlos a los estudios, hallazgos, análisis e interpretaciones de toda la investigación realizada, anterior y actual.

El hueso es un material arqueológico importante pero su conservación depende de las características del terreno, de la humedad y, en buena parte, de los valores de $\mathrm{pH}$ del terreno elegido para el Estudio. El pH constituye una medida del grado de acidez - ph inferior a 7-o alcalinidad - $\mathrm{pH}$ superior a 7 -. Parece demostrado que en terrenos muy ácidos, la duración de los restos óseos es menor. Por esta razón conviene realizar análisis del pH en el sitio arqueológico estudiado para conocer el grado de acidez o alcalinidad del terreno, con el estado de conservación que presenten las muestras óseas encontradas. También se presupone uno conocimiento adecuado de los "perfiles y clasificación de suelos".

Una primera impresión puede obtenerse con la observación, por examen directo, para identificar huesos humanos, describir anatómicamente el hueso descubierto y anotar las anormalidades si se presentan. Una información lo más completa posible, junto con las características y plano del sitio, con el lugar o lugares o de los hallazgos, para enviar todo al laboratorio.

En el entrenamiento del personal del campo que han de actuar en las excavasiones arqueológicas se contemplan nociones del esqueleto humano y rasgos más salientes para diferenciar los huesos humanos de otros mamíferos, una noción clara de la anatomía comparada. Estos conocimientos y trabajos se complementan con supervisiones permanentes que debieran ser hechas siempre por profesionales.

Un detalhe importante a tener en cuenta es que las muestras óseas seleccionadas para exámenes de mayor exactitud no deberán ser tratadas para conservación futura hasta que sean analizadas con las técnicas modernas citadas anteriormente. Cuando sea oportuno el tratamiento conservador es bueno recordar que ya no se aconseja la cera parafinada ni la goma laca, y sí resinas sintéticas, con lavado y secado previos.

Cuando en esos cementerios misioneros, los enterramientos continuaron despues de la expulsión de los jesuítas en 1768, e incluso hasta fechas relativamente recientes, es probable que en la "base" o en ciertos estratos, pueda identificarse un modo diferente del enterramiento jesuítico de la época misionera por algunas posiciones especiales y repetidas de esqueletos o 
restos óseos. La mayor problación concentrada repercutiría en un mayor número de enterramientos y como consecuencia en mayor número de muestras óseas. La experiencia obtenida en algunas excavaciones de las ruinas de estos pueblos misioneros ha permitido diferenciar tres períodos: pre-jesuítico, jusuítico misionero y posterior.

\section{Datación}

Varios de los pueblos misioneros jesuítico-guaraníes tuvieron más de una fecha de fundación porque fueron destruídos y reconstruídos o trasladados de lugar, con el mismo nombre. No obstante pueden ponerse ejemplos con los pueblos ya mencionados en el subtítulo de "Posibilidades de ejecución".

La primera fundación correponde a San Ignacio Guazú en 1609, hace 383 años, hoy en Paraguay, San Carlos en 1631, San Ignacio Mini en 1659 y Candelaria en 1665, en Argentina y San Miguel en 1687 y San Juan en 1697, en Brasil.

La fundación del pueblo misionero correspondería al primer año de utilización del cementerio.

En este Estudio podría marcarse una longitud cronológica entre los valores de 200 y 400 años.

En la datación de las muestras óseas de este Estudio, por ejemplo, el método de radiocarbono - C14 - tendría un valor muy relativo porque el margen de error generalmente llega a los 100 años y más. Es una prueba complicada y costosa, suministra datos menos precisos cuando se pretende obtener información de épocas más recientes. Es, exactamente, para los períodos históricos más cercanos cuando se exige una mayor precisión.

Simultáneamente con las excavaciones, en la sección vertical reciente del terreno, el geólogo o el ecólogo, puede "decidir" la edad de cada estrato o camada de tierra; dicho en mejores términos: con una secuencia geológica estratificada se logra una cronología de edades aproximadas.

Los métodos com el de fluorina, dendrocronología y otros, no estarían justificados en este Estudio porque las necesidades de este tipo de investigación se relaciona con la Arqueología Histórica, de épocas más recientes, y pretende ser una contribuición, un estudio complementario a la amplia investigación multidisciplinaria de las ruinas misioneras jusuítico-guaraníes. 
Con registros históricos y cartográficos, dignos de confianza, puede conseguirse una cronología válida que sería contrastada, si fuera necesario, con otros métodos y con los resultados de las investigaciones arqueológicas que son las que contribuyen de manera objetiva y científica, a demosntrar mejor la "realidad" del pasado misionero.

\section{Diagnóstico retospectivo}

Aunque el diagnóstico etiológico retrospectivo sea difícil, utilizando solamente la observación directa y cuidadosa del hueso en estudio, pueden plantearse hipótesis par dicutir las posibilidad más acertada que establezca un diagnóstico de sospecha. Lo expuesto se refiere exclusivamente al resultado del examen físico del hueso, pues si se despone de técnicas moderna de análisis, varias de ellas específicas, podrá obtenerse un resultado que indique la verdadera causa de la patología ósea encontrada.

El conocimiento previo de las características del esqueleto humano es indispensable. Si no se conocen los caracteres de los huesos normales no se pueden interpretar las anormalidades que se presenten ni diferenciarlos de otros mamíferos. Con frecuencia surgirán dudas que justifiquen el asesoramiento de un Profesor de Anatomía.

Conocida la organización, género de vida y alimentación de las misiones, es probable que exista concentración de huesos de ganado vacuno en lugares situados en las afueras de los pueblos y muestras procedentes de animales domésticos dentro de las áreas urbanas de los mismos.

Cuando se pone especial atención en los "resultados que se repiten" con iguales o parecidas características, condiciona un "sentido epidemiológico" que llevará a un diagnóstico de problemas de salud que padeció la comunidad que habitó el sitio o los sitios arqueológicos en estudio. Ello es más importante que el diagnóstico en un solo hueso o esqueleto, o en un solo espécimen de otro tipo de vestigios. En condiciones ideales, la investigación arqueológica en un cementerio com 200 a 400 añes de edad y población numerosa, como los de las misiones jesuítico-guaraníes, puede proporcionar un número adecuado de muestras para interpretar los resultados con sentido epidemiológico. Si esta condición no se apresenta habrá que conformarse con el estudio de las pocas muestras halladas.

En forma simplificada pueden establecerse algunos rasgos físicos anormales en los huesos que permitan pensar, inicialmente, en el diagnóstico de sospecha de una enfermedad determinada. No se trata de referir partes de 
un libro de Patología sino dar ideas y datos para motivar el interes por este tipo de investigaciones. El diagnóstico final de sospecha o de confirmación, será hecho siempre por especialistas, como resultado del examen físico directo en huesos y los análisis con las técnicas modernas mencionadas, hechas en huesos y en otros materiales o vestigios encontrados en las excavasiones - heces desecadas, por ejemplo-.

Tres llamadas de atención se consideran básicas, antes de formular cualquier primera impresión: a. las modificaciones que suelen originarse principalmente en huesos articulares, pasado cierto tiempo del enterramiento; b. los posibles aplastamientos que el esqueleto, o partes del mismo, hayan podido sufrir; y c. la corrosión, en diferentes grados, que puede ocorrir en los huesos por los efectos de una humedad excesiva y otras características negativas del terreno.

Otra precaución que debe estar presente antes de emitir un juicio diagnóstico es que generalmente las enfermedades infecciosas agudas y momentáneas o de corta duración, que son numerosas, rara vez dejan señales o lesiones óseas. Este conocimiento y experiencia determinan cierta cautela cuando se utilizan solamente los huesos en una investigación de la patología individual o coletiva en sitios arqueológicos.

La mortalidad en las poblaciones antiguas, incluyendo la de las misiones jesuítico-guaraníes, era alta y los índices por grupos de edad estaríam elevados con predominio de niños, jóvenes y adultos no muy mayores. El obtener resultados negativos o escasas lesiones en las muestras arqueológicas de huesos no quererá decir, siempre, que ese individuo o en esa comunidad no existían problemas de salud relacionadas con enfermedades infecciosas agudas, que serían probablemente la causa de una mortandad mayor. Es por esa razón que hoy debe recurrirse a otras pruebas o exámenes complementarios y modernos de diagnóstico.

¿En qué enfermedades podemos pensar cuando aparezcan anormalidades en huesos, completos o fragmentados, procedentes de excavasiones arqueológicas?

Los ejemplos, extraordinariamente simplificados, que despues se exponen darán la impresión de que el examen y su interpretación para llegar al diagnóstico, es tarea fácil. La realidad no es así. Se buscan signos o síntomas que generalmente son patognomónicos de una enfermedad, que sean característicos, que se presenten solamente en esa enfermedad. La frecuencia en su aparición ha que se les tome en cuenta pero no siempre ocurre así, además, que esos mismos signos pueden presentarse en otras enfermedades. Hay síntomas o signos que son más constantes en unas que 
en otras enfermedades. Por eso debe recurrirse a exámenes complementarios que aclaren las dudas y aproximen con cierta confianza al diagnóstico diferencial retrospectivo.

La extensa patología humana conocida hasta hoy no representa que toda las enfermedades repercutan en el sistema óseo originando problemas localizados o generalizados que ocasionan lesiones residuales a ser identificadas post-morten, pasados muchos años.

Enfermedades muy conocidas a través de los siglos - sífilis, tuberculosis, lepra y otras - sobre todo en su fase crónica y duración prolongada, ocasionan lesiones óseas que pueden persistir hasta la muerte del paciente y que son factibles de identificar posteriormente.

No solo algunas enfermedades infecciosas crónicas, como los ejemplos mencionados, pueden causar lesiones óseas, sino que otros trastornos tambiem originan este tipo de patología, tales como ciertas carencias de vitaminas y minerales - D y calcio, ocasionando raquitismo y osteomalacia -, procesos endocrinos, afecciones congénitas, tumores y caries.

Es probable que en un futuro no lejano, las tres enfermedade citadas, tuberculosis, sífilis, lepra y otras, tengan una rectificación en la clasificación donde siempre figuran como "importadas de Europa" y ello gracias a los métodos modernos de diagnóstico que se recomiendan en esta Contribuición.

Investigaciones de Arqueología Médica hechas en Chile, Perú, México y Estados Unidos y que constan en la bibliografía seleccionada, utilizando esos nuevos métodos y una evaluación histórica y arqueológica más afinadas, están demostrando la existencia pre-colombina, en las Américas, de diversas enfermedades que se creían importadas.

Un principio de diagnóstico médico y quirúrgico, válido en Arqueología Médica, es que "toda enfermedad ósea requiere una radiografía".

Tuberculosis ósea. Es más frecuente en niños y jóvenes. Deformación ósea. Las lesiones son principalmente del periostio. Decalcificación vertebral y hundimiento de algunas vértebras. La localización de los signos o síntomas de esta enfermedad se presenta en las extremidades de huesos largos, pequeños huesos de la mano y pies - la deformación fusiforme de los dedos de la mano es frecuente -. Cuando aparece en las vértebras, por haber padecido una espondilitis tuberculosa hay una deformación típica de la columna, con mayor localización en la región dorso-lumbar. La radigrafía, las pruebas serológicas y el examen histológico constituyen los mejores recursos para el diagnóstico y el diferencial con sífilis y otras enfermedades. 
Sífilis. Más frecuente en adultos y de origen perióstico, en forma de nódulos, generalmente múltiples. Se localizan principalmente en tibia, costilla, esternón, claviculas y cráneo. Cuando la sífilis ha sido un padecimiento muy prolongado, "periodo terciario" - y entonces no disponían de los tratamientos eficaces de hoy -, pueden parecer signos de osteomielitis destructivas y frecuentes caries seca. Su diagnóstico diferencial habría que hacerlo inicilmente con tuberculosis.

Lepra. Más frecuente en adultos, rara en niños e inexistente en menores de 3 años. Es una enfermedad de no fácil contagio y que tiene mucha historia. Los que sufrieron la forma anestésica, térmica y dolorosa, pueden presentar la amputación espontánea de los dedos, generalmente de la mano. Cuando aparezca esta lesión habrá que sospechar, inicialmente, en lepra aunque su diagnóstico diferencial con siringomielia y otras enfermedades es difícil, ya se trate de alteraciones en extremidades, mano, cráneo, arcada y piezas dentarias y otras partes del esqueleto.

Osteosis deformante. Enfermedad propia de la edad madur y senil. Se caracterza por aumento de la densidad ósea, espesamiento cortical, inclinación y crecimiento excesivo. Es recomendable hacer el diagnóstico diferencial con las metástasis ósea producidas por diversos tipos de cáncer, de mama o próstata.

Osteoporosis. Más común en mujeres menopáusicas que en hombres. Fracturas por impacto cón mínimos traumas - vértebras, pelvis y Colles -. Disminución de la densidad. En las radigrafías aparece el hueso com pérdida de la estructura trabecular.

Osteomielitis. - es la consecuencia de infección ósea. En las radiografías aparece destruicción ósea con secuestros.

Osteomalacia. - "Reblandecimiento" de los huesos. Deformidad causada por deficiencia de Vitamina D y desmineralización - calcio-. Produce raquitismo en niños y osteomalacia en adultos, originando deformaciones características. Se localiza principalmente en columna, pelvis y extremidades. Los huesos se curvan, en columna, pelvis y extremidades. La radiografía es un valioso soporte diagnóstico.

Neoplasias óseas. En patología ósea se dice que un aumento de volumen en un hueso hace sospechar un tumor hasta demostrar lo contrario. La formación de geodas de diferentes tamaños y zonas densas de calcificación contribuirán en la decisión diagnóstica. La observación directa, la radiografía y el examen histológico por microscopia de rutina o eletrónica aclararán el diagnóstico. 
Las fracturas producidas por traumatismos, y los callos que con frecuencia las acompañan, son de relativa facilidad diagnóstica.

Pruebas serológicas y $A D N$. Con técnicas modernas y confiables, hoy pueden diagnosticarse diversas enfermedades sin necesidad de recurrir al examen directo con microscopio para identificar el germen causante de un proceso patolológico. Este procedimiento contunúa siendo muy útil en la práctica diaria de los laboratorios clínicos, lo mismo en pesquisas médicas que arqueológicas, pero existen circunstancias en que su utilización no puede resolver el problema del diagnóstico requerido. Esta es la situación cuando se pretende manejar e examinar ciertos vestigios arqueológicos de origen humano para intenta llegar al diagnóstico de alguna enfermedades que habrían padecido los habitantes de los pueblos misioneros jesuítico-guaraníes durante los siglos XVII y XVIII.

Entre las nuevas técnicas con las que puede obtenerse diagnósticos por examen indirecto, resalta de la de "inmunodiagnóstico". Con el uso de las pruebas inmunológicas o serológicas, no se busca el diagnóstico de una enfermedad que padece actualmente un paciente sino que pudo haber tenido hace 100,200 o 400 años, manejando para ello vestigios o restos arqueológicos de procedencia humana. Claro que en la Clinica Médica se usa tambén para el diagnóstico "actual" de un enfermo. En el caso de este Estudio se utilizaría para el diagnóstico de una posible enfermedad "pasada". La prueba de inmunofluorescencia indirecta es probablemente la más usada. Tambén se utiliza la prueba inmunoenzimática conocida con el nombre de ELISA y otra en la que se marcan los antígenos con un isótopo radioactivo - $\mathrm{I}^{125}-$ denomidada IRMA; esta última presenta dificultades en la obtención de material radioactivo y en su eliminación una vez terminadas las pruebas.

Con las pruebas inmunológicas, usando antígenos específicos, es posible diagnosticar ciertas enfermedades, tales como tuberculosis, shigelosis - disentería bacilar -, amebiasis - disentería amebiana -, sífilis, toxiplasmosis, malaria, tripanosomiasis americana - enfermedad de Chagas -, leishmaniosis, triquinosis, schistosomiasis, salmonelosis - tifoidea y paratíficas - y otras.

La selección de antígenos específicos debería relacionarse con las posibilidades de adquirir antígenos de calidad y accesibles, y con los registros históricos donde ya existen datos que permitan diagnósticos retrospectivos de sospecha en las narraciones y descripciones de conquistadores, cronistas, historiadores y misioneros del Rio de La Plata, incluídos los pueblos misioneros jesuítico-guaraníes. 
El método más reciente es el Acido desoxirribonucleico o ADN que permite mayor seguridad en el diagnóstico. Esta prueba es uno de los brillantes resultados del progreso de la Biología Molecular.

\section{Exámenes radiológicos y microscópicos}

La radiografía es un valioso recurso técnico en la confirmación de un diagnóstico de enfermedad ósea en persona viva o en los restos óseos de material de arqueología. Por ser de fácil obtención en Clinicas Universitarias y Facultades de Medicina es un recurso que debiera aprovecharse al máximo, la radiografía y el radiólogo para la ejecución e interpretación de los resultados.

La microscopía adquiere suma importancia para el examen histopatológico del tejido óseo y la determinación de la edad. El examen microscópico también es un recurso de fácil accesibilidad en medios universitarios y resultará imprescindible para el exámen parasitológico.

A esos lugares detinados a cementerios es probable que se encuentren muestras que por su aspecto y ubucación las relacione con heces humanas en diferentes grados de desecación. Convendría tomar nota de los lugares donde algunos autores señalaron la instalación de letrinas, por ejemplo, en San Ignacio Guazú y en otros pueblos misioneros. En esta Contribuición no se cree necesario describir los métodos de rehidratación de la materia fecal que posibilita su exámen microscópico como si fueran recientes.

Con esas materias fecales rehidratadas y tratadas para examen parasitológico al microscopio, será posible detectar, si existieron huevos de helmintos intestinales, como por ejemplo, anqiolostomas y Trichiuris trichiura. Huevos de estos nematodes ya fueron encontrados en América del Sur, en coprolitos pre-colombinos de 1.000 y más años.

La identificación de materias fecales, su preparación para exámen microscópico e identificación de estos y otros helmintos, si existieron, será más fácil porque son muestras mucho más recientes. El éxito dependerá de la búsqueda cuidadosa y detallada que se realice.

En diversos registros históricos de los pueblos misioneros jusuíticoguaraníes, figuram como problema, las lombrices que los indios tenían en tal cantidad en su vientre que muchos morrían poe esa causa. También son frecuentes las referencias sobre las desinterías sangrientas - "cámaras" -. Si a esto añadimos la sospecha de la existencia de letrinas en las ruinas de 
algunos pueblos misioneros, se justificaría hacer sondeos representativos, también fuera de los cementerios.

\section{Grupos sanguíneos A, B, AB y O}

El grupo sanguíneo es un indicador objetivo, una característica de grupos humanos determinados, que persiste siempre, que es hereditario y que no se modifica por causas externas. Su determinación supone uma reacción serológica que titula anticuerpos. Es decisiva en Clínica Médica y Medicina Legal o Forense y adquiere singular importancia en Antropología y Arqueología.

En el caso específico del indio misionero guaraní, existen investigaciones que demuestran que más de $90 \%$ de los indio estudiados pertenecían al Grupo $\mathrm{O}$ y cuando hicieron el estudio en indios guaraníes puros que no se habían mezclado, dió el $100 \%$ del Grupo O. Estos estudios se realizaron entre los años 1934 y 1969, en comunidades guaraníes de Argentina, Brasil y Paraguay.

Estudios realizados en momias incaicas, pre-colombinas y coloniales de Chile y Perú demuestran que cerca del $90 \%$ tambén pertenecían al Grupo O.

Los resultados del estudio de los grupos sanguíneos en las investigaciones arqueológicas de los pueblos misioneros jesuítico-guaraníes permitirían compararlos con los resultados ya mencionados.

\section{Area de demostración}

Convendría que en cada país el Coordinador del Estudio designara un médico especialista en técnicas modernas de diagnóstico, quien se incorporaría al equipo encargado de realizar el trabajo de investigación. El médico, despues de conocer sus funciones, se comunicaría o reuniría con los médicos seleccionados por los otros dos países para tratar el Plan específico de ejecución, tomando en cuenta principalmente la uniformidad en procedencia de reactivos y antígenos y facilitar el desarrollo inicial de las técnicas, su aplicación e interpretación de resultados.

Como la investigación tiene un contenido multidisciplinario es indispensable que el médico tenga un conocimiento teórico y de trabajo de campo de la investigación arqueológica y así estar en condiciones de decidir, con 
los otros especialistas, su momento de actuar, de revisar la selección de muestra obtenidas y su preparación para transporte y examen. Aunque la mayor parte de su trabajo se desarrolla en laboratorio no le exime de participar en las reuniones y discusiones con los otros investigadores.

Si existieran dificultades para hacer este tipo de investigación de arqueología médica, simultáneamente en los tres países, podrían iniciarse individualmente e incorporarlas al Programa Anual de Investigaciones Arqueológicas en las ruinas de los pueblos misioneros jesuítico-guaraníes.

Un buen comienzo sería organizar una "Area de Demostración" donde fuera posible programar y poner a prueba la coordinación de actividades del médico y laboratorio dentro del equipo y fijar en detalle de la descripción y ejecución de sus funciones. Lo más apropiado sería que el médico seleccionado trabaje ya en un laboratorio clínico de calidad, dependiente de un Hospital Universitario y Facultad de Medicina.

El área de demostración puede considerarse como una etapa inicial y habrá que señalaria un período, dependiendo de las actividades arqueológicas que han de proporcionar las muestras para examen.

El país que comience los trabajos de arqueología médica podría transmitir sus primeras experiencias a los otros dos paises y organizar, y ofrecer, sus actividades de entrenamiento en su área de demostración.

La patología y lesiones que pueden encontrase en las excavaciones arqueológicas, así como las técnicas modernas de diagnóstico, representan un campa más amplio que el descrito. Aquí solamente se intenta da una orientación general y datos básicos que sirvan de estímulo para el comienzo de una investigación que será muy importante en el conocimiento de pasado misionero de los pueblos jesuítico-guaraníes y para prestigio de las Instituciones y de los investigadores que la realicen.

\section{BIBLIOGRAFIA BASICA Y ORIENTADORA}

BRAY, Warwick, TRUMP, David. Diccionario de arqueología. Madrid: Labor, 1976. RIBEIRO, Pedro Augusto Mentz. Manual de introdução à arqueologia. Porto Alegre: Sulina, 1977.

ALMAGRO, Martín. Introducción al estudio de la prehistoria y de la arqueología de campo. Madrid: Guadarrama, 1967.

COMAS, J. Manual de antropología física. México: Fondo de Cultura Económica, 1957. McINTOSH, Jane. Guía práctica de arqueología. Madrid: Herman Blume, 1987.

CHANG, K. C. Nuevas perspectivas en arqueología. Madrid: Alianza, 1966. 
KERN, Arno Alvarez. Problemas teórico-metodológicos relativos à análise do processo histórico missioneiro. In: Anais do VI Simpósio Nacional de Estudos Missioneiros. Santa Rosa: 1985.

PALACIOS, Silvio, ZÓFFOLI, Ena. Gloria y tragedia de las Misiones Guaraníes. Bilbao: Mensajero, 1991. p. 307-32.

FAIRBANK, T. Atlas of general affections of the skeleton. Edimburgo, 1951.

WEIS, Pedro. Osteología cultural. Lima (Peru): parte I, 1958; parte II, 1961.

HUBERLAKER, D. H. Human skeletal remains. Chicago: Aldine, 1978.

STEWARD, T. D. Pathological xhanges in South American indians skeletal remais. Bulletin of Snithsonian Institution American Ethn., 6:49-52, Washington, 1950.

WELLS, C. Bones, bodies and disease. London: Thames \& Hudson, 1964.

BROTWEL, D. R. Digging up bones. The excavation, treatment and study of human skeletal remains. London: British Museun Publications, 1981.

CORNWALL, I. Bones for the archeologist. London: 1954.

STENBOCK, R. Paleopathological diagnosis and interpretation. Springfield: Thomas, 1976.

ALISON, M. J. et al. Tuberculosis in pre-columbian andean populations. Illinois: Evanston, 1981. Jane E. Binkstra Ed. Northwestern University Archeological Program.

ROTHHAMMER, F. Chagas disease in chilean mummies. Parasitology Today, v. 1, n. $5,1985$.

ALLISON, M. J. Pre-columbian american disease. Research Reports, v. 12, 1971; v. 18, 1980. Virginia University, Richmon, Virginia. National Geographic Society, Washington.

BROTHWEL, D. R., HIGGS, E. Ciencia en arqueología; la aplicación de los rayos $X$ en el estudio de material arqeológico. México: Fondo de Cultura Económica, 1980.

SAUL, Frank P. Life history as recorded in maya skeleton fron Cozumel. Research Reports, v. 20, 1985. Medical College of Ohio; National Geographic Society, Washington.

ANAIS do Segundo Simpósio de Arqueologia da área do Prata. Pesquisas, Antropologia, n. 18. Estudos Leopoldenses, n. 9; Instituto Anchietano de Pesquisas, 1968.

CONTRIBUIÇÃO às pesquisas arqueológicas através de análises osteológicas. IV Reuniāo Científica da Sociedade de Arqueologia Brasileira - SAB. Santos, 1987.

ARAUJO, Adauto José Gonçalves de. Contribuição ao estudo de helmintos encontrados em material arqueológico no Brasil. Rio de Janeiro: Instituto Osvaldo Cruz, 1980.

BANDEIRA, Carlos Manes, GÓMES, Luiz Octavio Louro. Arqueologia histórica. Pesquisas 4, Fundação Brasileira para a Conservação da Natureza, 1978.

CANDELA, P. B. Blood groups reactions in ancient human skeletons. American Journal Phys. Anthropology, Washington, 1936.

THE blood groups of the ancient dead. Science, Washington, 1960.

KOUT, M. et al. An attemp to asses groups in paleoanthropological material. Anthropologie, 2:49-58, Paris. 


\section{APENDICE}

Organismos nacionales e internacionales y personas para consulta y/o ejecución de este Proyecto.

\section{Argentina}

Ministerio de Cultura y Educación, Secretaria de Cultura, Comisión Nacional de Museos, Monumentos y Lugares Históricos, Arquitecto Dr. Jorge Hardoy, Avenida de Mayo, $\mathrm{n}^{2} 556$, Buenos Aires.

Dra. Elsa Segura, Instituto Satala Chabed, Paseo Colón, $n^{0} 563$, Buenos Aires.

Universidad Nacional del Nordeste, Professor Arquitecto Ramón Gutierrez, Facultad de Arquitectura y Urbanismo. Professor Ernesto J. A. Maeder, Director de Investigaciones Geohistóricas, Resistencia, Chaco.

Instituto "Ruiz de Montoya", Dra. Mary Hadad, Directora del Centro de Interpretación Jesuítico-Guaraní. Arqueóloga. Licenciada Ruth Adela Pujade, Posadas, Missiones.

\section{Brasil}

Ministério de Cultura, Instituto Brasileiro do Patrimônio Cultural IBPC - Presidente Dra. Lelia Cotijo Suáres, Rio de Janeiro.

Regional do IBPC, Arquiteto Luiz Antonio Volcato Custodio, Porto Alegre, RS.

Pontifícia Universidade Católica do Rio Grande do Sul - PUCRS -, Curso de Pós-Graduação em História, Arqueólogo e Historiador Professor Dr. Arno Alvarez Kern, Porto Alegre, RS.

Faculdade de medicina, Porto Alegre, RS.

Centro de Biotecnologia do Rio Grande do Sul, Dr. Luiz Shozo Ozaki, orientación para inmunodiagnóstico y DNA.

Ministério da Saúde, Fundação Osvaldo Cruz, Escola Nacional de Saúde Pública, Laboratório de Paleoparasitologia, Dr. Luiz Fernando Ferreira y Dr. U. Confalonieri (tienen muy buenos trabajos con técnicas de examen y resultados en muestras de coprolitos procedentes de sitios arqueológicos de Brasil y del exterior). Rio de Janeiro, RJ. 


\section{Paraguay}

Ministerio de Obras Públicas y Comunicaciones, Dirección General de Turismo, Dr. Derlis Esteche, Palma, $n^{2} 470$, Asunción.

ICOMOS, Arquitecto Jorge Platiño, Asunción

Universidad de Nuestra Señora de la Asunción, Centro de Estudios Antropológicos, Dr. Bartolomeu Meliá, S. J., Asunción.

PARACUARIA, Fundación Privada de Alemania, Consulado General de Alemania, Asunción.

Facultad de Medicina, Asunción.

\section{Organismos Internacionales}

Organización de las Naciones Unidas para la Educación, la Ciencia y la Cultura - UNESCO -, 7 Place de Fontenoy, 757000, París, Francia, Director General Professor Dr. Federico Mayor Zaragoza.

Instituto de Cooperación Iberoamericana, Avenida de los Reyes Católicos, $n^{0} 4-28040$, Madrid, España, Director General Dr. Francisco Javier Jiménez-Ugarte Hernández.

\section{Referencias que pueden ser útiles en estas investigaciones}

En los tres países incluídos, Argentina, Brasil y Paraguay, será importante consultar bibliografía específica de pesquisas arqueológicas relacionadas con la medicina y de trabajos de la misma índole, presentados en las reuniones científicas de las Sociedades de Arqueología.

Dr. T. Dale Stewart, Phisical Antropologist of Smithsonian Institution, South Side at 10th S.W. 20560 - Washington, D.C., USA.

Dr. Winfield Swanson, National Geographic Research, National Geographic Society, 1600 M Street, N.W. 20013 - 1640 - Washington, D.C., USA.

Center for Desease Control - CDC -, Public Health Service, national Institute of Helath, Atlanta, Georgia, USA. El CDC podría ser consultado sobre antígenos y servir de posible Laboratorio de Referencia.

Las Instituciones mencionadas suelen otorgar facilidades para trabajos "originales" de investigación, dependiendo del enfoque científico y técnico 
del Proyeto, Instituiciones y personal calificado que interviene, esposición detallada de objetivos y metodología, repercusión de sus resultados, tiempo de ejecución y coste total con la justificación de la ayuda técnica y/o financiera solicitada. 